\title{
Three-parameter Lorentz-violating texture for neutrino mixing
}

\author{
Jorge S. Díaz, V. Alan Kostelecký* \\ Physics Department, Indiana University, Bloomington, IN 47405, USA
}

\section{A R T I C L E I N F O}

\section{Article history:}

Received 17 February 2011

Received in revised form 22 April 2011

Accepted 23 April 2011

Available online 29 April 2011

Editor: A. Ringwald

\section{Keywords:}

Lorentz violation

CPT violation

Neutrino oscillations

\section{A B S T R A C T}

A three-parameter model of neutrino oscillations based on a simple Lorentz- and CPT-violating texture is presented. The model is consistent with established data and naturally generates low-energy and neutrino-antineutrino anomalies of the MiniBooNE type. A one-parameter extension incorporates the MINOS anomaly, while a simple texture enhancement accommodates the LSND signal.

(C) 2011 Elsevier B.V. All rights reserved.
In the minimal Standard Model (SM) of particle physics, the three types of neutrinos are massless and preserve flavor as they propagate. However, compelling evidence now exists for neutrino flavor oscillations, including the confirmed disappearance of solar, reactor, atmospheric, and accelerator neutrinos. The canonical explanation for these oscillations assumes that the three known flavors of neutrinos have a tiny mass matrix with nondiagonal components. In the usual extension of the SM to three flavors of massive neutrinos $(3 v \mathrm{SM})$, the $3 \times 3$ matrix governing oscillations involves two mass-squared differences, three mixing angles, and a $\mathrm{CP}$-violating phase. For suitable values of these six parameters, the $3 v$ SM successfully describes established oscillation data [1].

In recent years, several experiments have adduced some evidence for anomalous neutrino oscillations that cannot be accommodated in the $3 v \mathrm{SM}$. These include the LSND signal [2], the MiniBooNE low-energy excess [3], and neutrino-antineutrino differences in the MiniBooNE [4] and MINOS [5] experiments. No satisfactory global description of these anomalies exists to date. Here, we focus on the possibility that Lorentz and CPT violation could be responsible for a substantial part of the existing oscillation data, including some or all of the anomalies.

Observable effects of Lorentz and CPT violation are conveniently described by effective field theory [6]. Experimental data can be analyzed using the Standard-Model Extension (SME) [7], which is the comprehensive realistic framework for Lorentz violation containing the SM and General Relativity and incorporating CPT vio-

\footnotetext{
* Corresponding author.

E-mail address: kostelec@indiana.edu (V.A. Kostelecký).
}

lation [8]. Numerous searches for nonzero coefficients for Lorentz and CPT violation have been undertaken in recent years using a broad range of methods [9]. In the SME context, the phenomenology of neutrino oscillations [10-22] and the development of techniques to extract limits from short- and long-baseline experiments [23] have stimulated several experimental analyses [24-28].

In this work, we study a simple texture $h_{\mathrm{eff}}^{v}$ for the $3 \times 3$ matrix governing oscillations of three flavors of active left-handed neutrinos that involves isotropic Lorentz and CPT violation in a chosen frame. This requires breaking boost symmetry, which implies neutrino mixing acquires nonstandard dependence on the neutrino energy $E$. In contrast to mass terms, which permit only a $1 / E$ dependence, isotropic Lorentz violation from effective field theory introduces nonstandard energy dependence even in vacuum oscillations, a unique signal.

The texture $h_{\mathrm{eff}}^{v}$, which we call the 'puma' model, was discovered by a systematic hunt through the jungle of possible SMEbased models. Among other criteria, candidate textures were required to have a simple analytical form involving no more than three parameters. Both mass terms and Lorentz-violating operators of arbitrary dimension [29] were included in the analysis. The various candidates were vetted by requiring compatibility with all compelling oscillation data. Here, we present an interesting model that describes established oscillation data with only three parameters instead of the six in the 3vSM. Remarkably, this model also naturally reproduces the two MiniBooNE anomalies without extra degrees of freedom, a feature manifestly impossible to achieve by adding to the $3 v \mathrm{SM}$ more neutrinos or unconventional interactions. Moreover, comparatively simple enhancements can accommodate the LSND signal and the MINOS anomaly. The results presented here suggest that Lorentz- and CPT-violating models offer 
a valuable direction to pursue in searches for simple but realistic neutrino-mixing textures.

For neutrinos, the $3 \times 3$ texture $h_{\mathrm{eff}}^{v}$ can be written in the flavor basis and in the isotropic frame as

$h_{\mathrm{eff}}^{v}=A\left(\begin{array}{lll}1 & 1 & 1 \\ 1 & 1 & 1 \\ 1 & 1 & 1\end{array}\right)+B\left(\begin{array}{lll}1 & 1 & 1 \\ 1 & 0 & 0 \\ 1 & 0 & 0\end{array}\right)+C\left(\begin{array}{lll}1 & 0 & 0 \\ 0 & 0 & 0 \\ 0 & 0 & 0\end{array}\right)$,

where $A(E)=m^{2} / 2 E, B(E)=\stackrel{\circ}{a} E^{2}$, and $C(E)=\stackrel{\circ}{c} E^{5}$ are real and exhibit a simple energy dependence. Note that this texture involves only one neutrino mass parameter $m$. In the SME, the coefficients $\stackrel{\circ}{a}$ and $\stackrel{\circ}{c}$ control operators for isotropic Lorentz violation of dimension five and eight, respectively. The operator for $\stackrel{\circ}{a}$ also breaks CPT, so the $3 \times 3$ matrix $h_{\text {eff }}^{\bar{v}}$ governing antineutrino mixing is obtained by reversing the sign of $\stackrel{\circ}{a}$ while keeping $m$ and $\stackrel{\circ}{c}$ unchanged. Since $h_{\text {eff }}^{v}$ is $T$ invariant, $\stackrel{\circ}{a}$ also determines CP-violating effects. For definiteness, in this work we fix $m^{2}=2.6 \times 10^{-23} \mathrm{GeV}^{2}, \stackrel{\circ}{a}=-2.5 \times$ $10^{-19} \mathrm{GeV}^{-1}$, and $\stackrel{\circ}{c}=1.0 \times 10^{-16} \mathrm{GeV}^{-4}$, but a range of other choices also yields reasonable agreement with data. The value for $m$ is consistent with limits from direct mass measurements and cosmological bounds [1]. Intriguingly, the texture (1) is uniformly populated at low energies, while higher energies reveal Lorentzviolating electron-flavor corrections that might emerge from a unified theory at the Planck scale [30].

Inspection reveals that $h_{\text {eff }}^{v}$ has a vanishing eigenvalue. As a result, many calculations reported here can be performed analytically with comparative ease. The eigenvalues $\lambda_{1} \equiv \lambda_{-}, \lambda_{2} \equiv \lambda_{+}, \lambda_{3} \equiv 0$ of $h_{\mathrm{eff}}^{v}$ are readily found by diagonalization via a unitary mixing matrix $U(E)$,

$\lambda_{ \pm}=\frac{1}{2}\left[3 A+B+C \pm \sqrt{(A-B-C)^{2}+8(A+B)^{2}}\right]$.

The eigenvalues and mixing angles have nontrivial energy dependence, a feature absent in the $3 \nu \mathrm{SM}$. The oscillation probability $P_{v_{a} \rightarrow v_{b}}(E)$ between flavor states $a, b$ has an amplitude formed from products of $U(E)$ and a phase determined by the dimensionless product of the baseline $L$ and the eigenvalue differences $\Delta_{a^{\prime} b^{\prime}}(E)=\lambda_{a^{\prime}}-\lambda_{b^{\prime}}$ between eigenstates $a^{\prime}, b^{\prime}$ [10]. The oscillation lengths $L_{a^{\prime} b^{\prime}}(E) \equiv 2 \pi / \Delta_{a^{\prime} b^{\prime}}(E)$ of the model (1) have more complicated energy dependence than those of the $3 v \mathrm{SM}$, which grow linearly with energy. Since $h_{\text {eff }}^{v}$ and $h_{\text {eff }}^{\bar{v}}$ differ by the sign of $\stackrel{\circ}{a}$, so do the antineutrino mixing matrix $\bar{U}(E)$ and oscillation lengths $\bar{L}_{a^{\prime} b^{\prime}}(E)$.

At low energies, the matrix (1) is dominated by the mass parameter. As a result, the phase of the oscillation probability becomes proportional to $m^{2} L / E$, matching the behavior in the $3 v \mathrm{SM}$. The texture becomes democratic and exhibits $S_{3}$ invariance, so the neutrino flavors are tribimaximally mixed. This guarantees consistency of the model both with the KamLAND observation of longbaseline reactor-antineutrino disappearance [31] and also with the disappearance of neutrinos in the low-energy region of the solarneutrino spectrum [32]. For solar neutrinos, adding to $h_{\mathrm{eff}}^{v}$ the usual effects from forward scattering in the Sun yields results consistent with solar data and the 3vSM prediction [33]. Differences arise at energies above $30 \mathrm{MeV}$, which lie beyond the threshold of the solar-neutrino spectrum. In effect, the model eliminates the $3 v$ SM solar mixing angle $\theta_{12}$ as a degree of freedom, with the observed mixing reproduced as a direct consequence of the democratic structure of the texture (1) at low energies.

At high energies, the mass term $A$ in $h_{\text {eff }}^{v}$ becomes negligible. The size and energy dependences of the terms $B$ and $C$ trigger a Lorentz-violating seesaw mechanism [10] that makes the eigenvalue $\lambda_{-}$proportional to $\stackrel{\circ}{ }^{2} / \stackrel{\circ}{c} E$. The oscillation phase becomes proportional to $L / E$, while the combination $\stackrel{\circ}{a}^{2} /{ }^{\circ}$ behaves like an
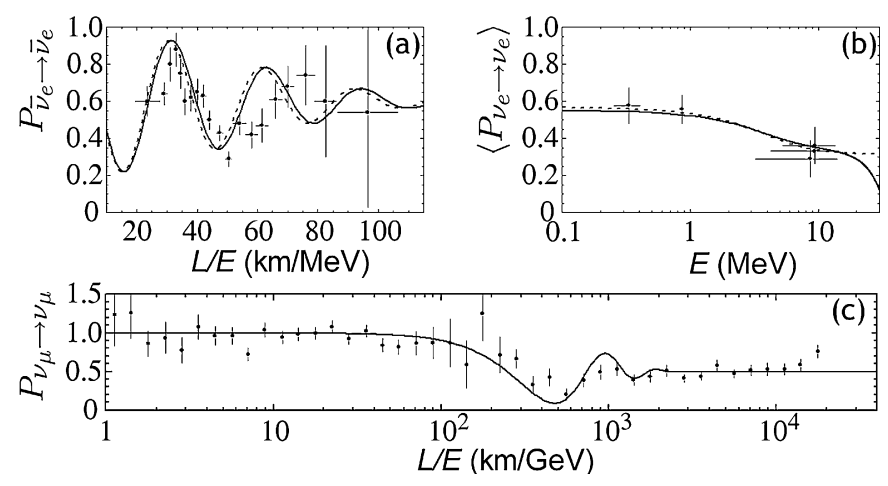

Fig. 1. Puma model (solid) and 3vSM (dashed) compared to (a) KamLAND [31], (b) solar [33], and (c) SK [34] data.

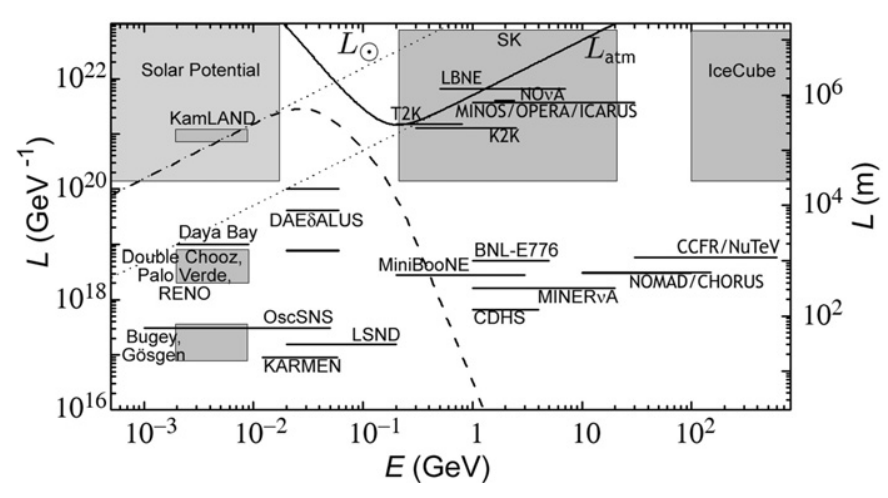

Fig. 2. Energy dependence of oscillation lengths in the puma model.

effective mass. The contributions from $B$ and $C$ break the lowenergy $S_{3}$ symmetry to the $S_{2}$ subgroup in the $\mu-\tau$ sector. As a result, one eigenstate is a uniform mixture of the $\mu$ and $\tau$ flavors, which produces maximal mixing for atmospheric neutrinos in agreement with observed results [34]. The form of the texture (1) therefore also eliminates the 3vSM atmospheric mixing angle $\theta_{23}$ as a degree of freedom.

Fig. 1 compares oscillation probabilities obtained from $h_{\mathrm{eff}}^{v}$ (solid lines) and from the 3vSM (dashed lines) with KamLAND, solar-neutrino, and Super-Kamiokande (SK) data. The simple texture (1) provides a remarkable match. This accomplishment requires only two of the three degrees of freedom in the model, as described above. Achieving comparable results in the $3 v \mathrm{SM}$ involves four of its six degrees of freedom instead.

Plotting a given oscillation length as a function of the energy $E$ together with the relevant experimental coverage in $E-L$ space offers a powerful visual guide to oscillation signals [10]. Provided the relevant oscillation amplitude is appreciable, neutrino oscillations are significant in the region above the curve but are mostly negligible below it. Fig. 2 displays the $E-L$ coverage of several experiments and shows the antineutrino oscillation lengths $\bar{L}_{a^{\prime} b^{\prime}}(E)$ from $h_{\mathrm{eff}}^{\bar{v}}\left(\bar{L}_{31}\right.$, solid curve; $\bar{L}_{21}$, dashed curve) and from the $3 v \mathrm{SM}$ ( $L_{\odot}$ and $L_{\mathrm{atm}}$, dotted straight lines). The figure reveals that the simple texture (1) yields low-energy reactor oscillations controlled by $\bar{L}_{21}$, which has the $3 \nu \mathrm{SM}$ oscillation length $L_{\odot} \propto E / \Delta m_{\odot}^{2}$ as an asymptote. Also, high-energy atmospheric oscillations are determined by $\bar{L}_{31}$, which approaches the $3 v$ SM oscillation length $L_{\mathrm{atm}} \propto E / \Delta m_{\mathrm{atm}}^{2}$. The corresponding plot for neutrinos is similar overall, with differences arising from the sign change of $\stackrel{\circ}{a}$ appearing primarily in the region $10-100 \mathrm{MeV}$.

Fig. 2 also reveals that the texture (1) is compatible with the null data for antineutrino disappearance from short-baseline reactor experiments. The baseline $L$ for these experiments lies well 
below the $\bar{L}_{21}$ curve, so no oscillations occur even though the relevant components of the mixing matrix $U(E)$ are large. In contrast, the $3 \nu \mathrm{SM}$ accounts for these experimental results as a consequence of a small mixing angle $\theta_{13}$. The texture (1) is also consistent with null oscillation results reported by short-baseline accelerator experiments at high energies $\gtrsim 1 \mathrm{GeV}$. For many of these, the mixing angles in $U(E)$ essentially vanish above $500 \mathrm{MeV}$. Large mixing angles in $U(E)$ appear for a subset of experiments studying $v_{\mu} \rightarrow v_{\tau}$. However, no mixing is predicted because this oscillation channel is controlled by $L_{31}$, which lies far above this region in $E-L$ space.

Since the texture (1) is Lorentz violating, anisotropies must appear in any boosted frame. Boosts of relevance to Earth-based experiments include the solar velocity $\beta \simeq 10^{-3}$ relative to the cosmic microwave background, the Earth's revolution velocity $\beta \simeq$ $10^{-4}$ about the Sun, and the tangential velocity $\beta \simeq 10^{-5}$ of a laboratory rotating with the Earth. These boosts generate tiny anisotropic contributions to $h_{\mathrm{eff}}^{v}$ and $h_{\mathrm{eff}}^{\bar{v}}$ that among other effects imply sidereal variations of oscillations in the laboratory frame [35]. In particular, boosting $B$ introduces anisotropies in all the $e$ components, while boosting $C$ introduces ones in the ee component. Of these, only the $e \mu$ components are experimentally constrained to date [24-26]. An analysis reveals that the predicted signals from $h_{\text {eff }}^{v}$ remain a factor of $10-100$ below the attained experimental sensitivity, even for the maximal boost $\beta \simeq 10^{-3}$.

Taken together, the above results indicate that the matrix $h_{\mathrm{eff}}^{v}$ is compatible with confirmed experimental data at all energies. This match is achieved using only three real parameters, of which two degrees of freedom are fixed by the existing data from accelerator experiments and from solar- and atmospheric-neutrino measurements. However, the predictions of the texture (1) and of the $3 v$ SM differ significantly in the range $10 \mathrm{MeV} \lesssim E \lesssim 1 \mathrm{GeV}$, as can be seen in Fig. 2. One pleasant surprise is that at high energies the small Lorentz-seesaw eigenvalue proportional to $1 / E$ must be accompanied by a large eigenvalue growing rapidly with $E$. This naturally enforces a steep drop with energy of the length $L_{21}$ relevant for $v_{\mu} \rightarrow v_{e}$ oscillations. Our value for the third degree of freedom permits $L_{21}$ to pass through the region of sensitivity of the MiniBooNE experiment. Since the oscillation amplitude is large and decreases rapidly with energy in the same region, a signal is generated at energies 200-500 MeV. Moreover, CPT violation involving $\stackrel{\circ}{a}$ makes the oscillation signal greater for neutrinos than antineutrinos. Evidence for both these features is reported in the MiniBooNE data $[3,4]$. We emphasize that these features emerge from the texture (1) without additional particles, forces, or degrees of freedom. Fig. 3 shows results from $h_{\mathrm{eff}}^{v}, h_{\mathrm{eff}}^{\bar{v}}$, the $3 \nu \mathrm{SM}$, and the tandem model [36], which predicted a small low-energy excess prior to its discovery. For both neutrinos and antineutrinos, $h_{\text {eff }}^{v}$ and $h_{\text {eff }}^{\bar{v}}$ provide a better match using a simple $\chi^{2}$ statistic per degree of freedom. They also improve over the 'best fits' obtained by varying non-3vSM values of $\Delta m^{2}$ and $\sin ^{2} 2 \theta$ independently for neutrinos $\left(\chi_{v}^{2}=2.6\right)[3]$ and antineutrinos $\left(\chi_{\bar{v}}^{2}=1.1\right)[4]$.

Several forthcoming experiments using reactor antineutrinos and long-baseline accelerator neutrinos are being designed to obtain precise measurements of the $3 v \mathrm{SM}$ mixing angle $\theta_{13}$ for CPTinvariant $\mathrm{CP}$ violation. Among the experiments involving reactorantineutrino disappearance [37], most are insensitive to oscillations arising from $h_{\text {eff }}^{\bar{v}}$ because they are located well beneath the $\bar{L}_{21}$ curve in Fig. 2. Analysis reveals that the largest signal from $h_{\mathrm{eff}}^{\bar{v}}$ appears in the Daya Bay experiment in the region of $2-3 \mathrm{MeV}$, where the $3 v \mathrm{SM}$ predicts no oscillations. Other experiments plan to investigate $v_{\mu} \rightarrow v_{e}$ and $\bar{v}_{\mu} \rightarrow \bar{v}_{e}$ transitions using long baselines of several hundred kilometers [38], for which the oscillation amplitude associated with $h_{\mathrm{eff}}^{v}$ decreases rapidly above $500 \mathrm{MeV}$.

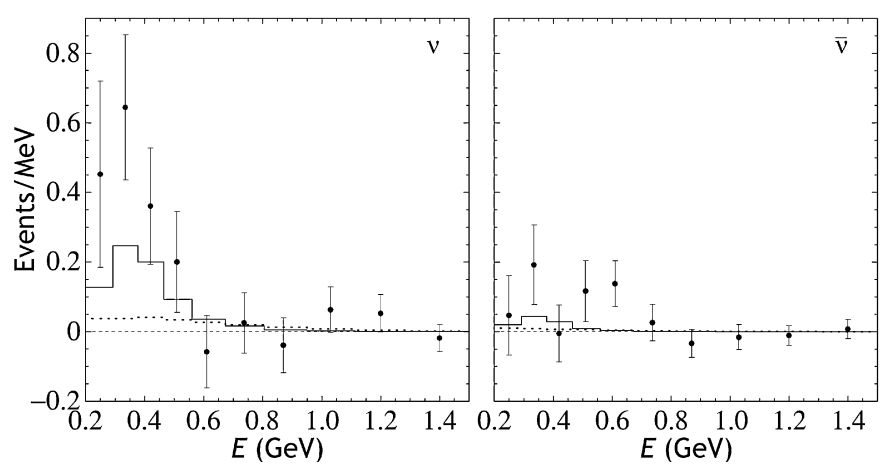

Fig. 3. Comparison of the puma model (solid lines; $\chi_{\nu}^{2}=1.0, \chi_{\bar{v}}^{2}=0.9$ ), the tandem model [36] (dotted lines; $\chi_{v}^{2}=1.9, \chi_{v}^{2}=1.0$ ), and the 3vSM (dashed lines; $\chi_{v}^{2}=$ 2.2, $\chi_{\bar{v}}^{2}=1.1$ ), with MiniBooNE neutrino [3] and antineutrino [4] data.

The largest oscillation signal from $h_{\mathrm{eff}}^{v}$ in this group is about $2 \%$ near $300 \mathrm{MeV}$ in the T2K experiment, where the $3 v \mathrm{SM}$ signal is below $0.5 \%$. Substantial CPT violation also appears in the range 200-300 MeV, with the $v_{\mu}$ survival probability reaching a zero minimum while the $\bar{v}_{\mu}$ survival remains above 0.8 . Another proposal is the DAE $\delta A L U S$ experiment [39], which has a common detector for three $\bar{v}_{\mu}$ sources with different baselines. For the currently proposed baselines, the model (1) predicts signals an order of magnitude or more lower than the $3 v \mathrm{SM}$. However, at a baseline of about $100 \mathrm{~km}, h_{\mathrm{eff}}^{\bar{v}}$ produces a large oscillation signal that grows with energy, where the $3 v$ SM signal decreases with energy instead.

The effectiveness of the texture (1) in reproducing established data and satisfying constraints suggests it offers an interesting basis from which to attempt the construction of a model that also describes the antineutrino-oscillation anomalies reported by the LSND [2] and MINOS [5] experiments. One simple texturepreserving extension of the three-parameter model involves an additional contribution to the $e-\mu$ and $e-\tau$ sectors of the form ${ }^{\prime}{ }^{\prime} E$, where $\stackrel{\circ}{c}^{\prime}$ is a coefficient for CPT-even Lorentz violation chosen here for definiteness as ${ }^{\prime} c^{\prime}=2.0 \times 10^{-20}$. The presence of this fourth degree of freedom leaves unaffected the main features of the three-parameter model but combines with the coefficient $\stackrel{a}{a}$ to trigger differing oscillation probabilities for neutrinos and antineutrinos at high energies. The difference appears as a relative shift between the minima of the survival probabilities for neutrinos and antineutrinos. This is consistent with the MINOS anomaly, as can be seen in Fig. 4. Similar shifts are also predicted in future longbaseline accelerator experiments [38], with a notable peak in the $\mathrm{T} 2 \mathrm{~K}$ antineutrino data where the $3 \nu \mathrm{SM}$ predicts a minimum. We emphasize that these CPT-violating effects are achieved without neutrino-antineutrino mass differences and are consistent with effective field theory [8]. Indeed, the only mass parameter in the model appears in the original texture $h_{\mathrm{eff}}^{v}$, and it is negligible at MINOS energies.

The LSND signal [2] is absent from the three-parameter model $h_{\text {eff }}^{\bar{v}}$ because the baseline lies far below the $\bar{L}_{21}$ curve in Fig. 2 . However, another option for enhancing the model is an energylocalized modification that preserves compatibility of the texture with established data. We consider here a simple three-parameter gaussian enhancement $\delta h_{\mathrm{eff}}^{\bar{v}}=\alpha \exp \left[-\beta(E-\epsilon)^{2}\right]$ for the $\bar{e}-\bar{\mu}$ and $\bar{e}-\bar{\tau}$ sectors of $h_{\text {eff }}^{\bar{v}}$. The CPT-conjugate enhancement is obtained by changing the signs of $\alpha$ and $\epsilon$, which maps its effects outside the physical range and so leaves neutrino oscillations unaffected. Introducing an enhancement $\delta h_{\text {eff }}^{\bar{v}}$ can produce a localized valley in the $\bar{L}_{21}$ curve with minimum approaching the region of LSND sensitivity. For example, Fig. 4 shows the signal from a $\delta h_{\mathrm{eff}}^{\bar{v}}$ centered 

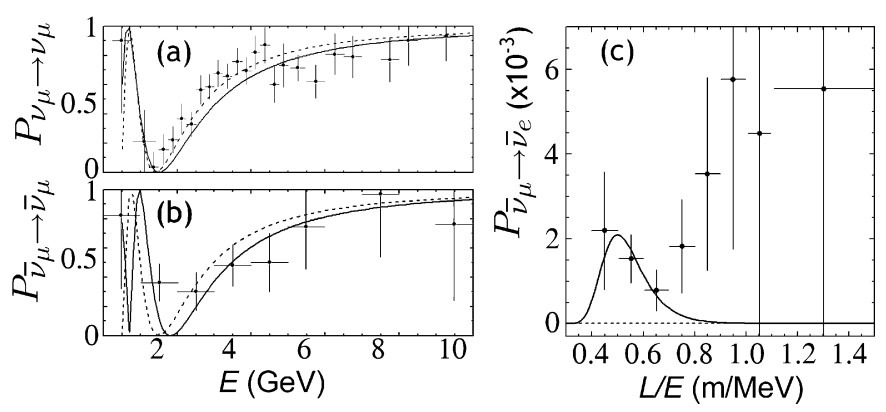

Fig. 4. Comparison of texture enhancements of the puma model (solid lines; $\chi_{v}^{2}=$ $1.4, \chi_{\bar{v}}^{2}=0.9, \chi_{\mathrm{LSND}}^{2}=1.6$ ) and the $3 \nu \mathrm{SM}$ (dashed lines; $\chi_{v}^{2}=1.0, \chi_{\bar{v}}^{2}=1.6$, $\chi_{\text {LSND }}^{2}=2.6$ ) with (a) MINOS neutrino data [34], (b) MINOS antineutrino data [5], and (c) LSND antineutrino data [3].

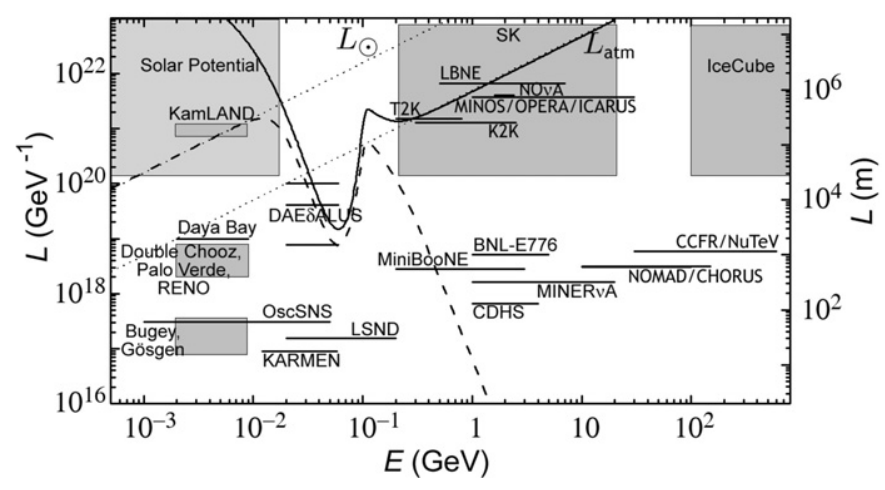

Fig. 5. Energy dependence of oscillation lengths in the doubly enhanced puma model.

at $\epsilon=60 \mathrm{MeV}$ with amplitude $\alpha=3.0 \times 10^{-19} \mathrm{GeV}$ and width $\beta=3.0 \times 10^{3} \mathrm{GeV}^{-2}$. No effects arise in other existing experiments because the valley is localized in $E$, while sidereal variations lie below current limits. However, this enhancement predicts a nonzero probability for the planned OscSNS experiment [40], growing to about $0.5 \%$ at $50 \mathrm{MeV}$. It also predicts large effects from all three sources in the proposed DAE $\delta$ ALUS experiment [39], with a striking signal from the near source that is about 100 times larger than the $3 v$ SM expectation.

The above extension and enhancement lie in disjoint energy regions, so we can incorporate both simultaneously. The result is a seven-parameter doubly enhanced texture that appears globally compatible with all compelling data and the various existing anomalies. The changes to the eigenvalues (2) of $h_{\text {eff }}^{v}$ arising from the double enhancement are found by the substitutions $B \rightarrow B+\delta h, C \rightarrow C-\delta h$ with $\delta h=\dot{c}^{\prime} E-\alpha \exp \left[-\beta(E+\epsilon)^{2}\right]$. The effect on the oscillation lengths is shown in Fig. 5. Since the anomalies remain to be confirmed experimentally and since the doubly enhanced texture requires four more parameters than the frugal three of $h_{\mathrm{eff}}^{v}$, we interpret this construction primarily as an existence proof revealing the surprising effectiveness of simple Lorentz-violating textures. However, any putative enhancement of the $3 v \mathrm{SM}$ will also require extra degrees of freedom, and the seven-parameter texture does appear at present to offer the only global description incorporating all anomalies. Further tests of these ideas can be expected in the near future from additional experimental data elucidating the various anomalies.

\section{Acknowledgements}

This work was supported in part by the Department of Energy under grant DE-FG02-91ER40661 and by the Indiana University Center for Spacetime Symmetries.

\section{References}

[1] K. Nakamura, et al., J. Phys. G 37 (2010) 075021.

[2] A. Aguilar, et al., Phys. Rev. D 64 (2001) 112007.

[3] A.A. Aguilar-Arevalo, et al., Phys. Rev. Lett. 102 (2009) 101802.

[4] A.A. Aguilar-Arevalo, et al., Phys. Rev. Lett. 105 (2010) 181801.

[5] P. Vahle, Nucl. Phys. B (Proc. Suppl.), in press.

[6] V.A. Kostelecký, R. Potting, Phys. Rev. D 51 (1995) 3923.

[7] D. Colladay, V.A. Kostelecký, Phys. Rev. D 55 (1997) 6760; D. Colladay, V.A. Kostelecký, Phys. Rev. D 58 (1998) 116002; V.A. Kostelecký, Phys. Rev. D 69 (2004) 105009.

[8] O.W. Greenberg, Phys. Rev. Lett. 89 (2002) 231602.

[9] V.A. Kostelecký, N. Russell, Rev. Mod. Phys. 83 (2011) 11.

[10] V.A. Kostelecký, M. Mewes, Phys. Rev. D 69 (2004) 016005; V.A. Kostelecký, M. Mewes, Phys. Rev. D 70 (2004) 031902(R).

[11] V. Barger, D. Marfatia, K. Whisnant, Phys. Lett. B 653 (2007) 267.

[12] N. Cipriano Ribeiro, et al., Phys. Rev. D 77 (2008) 073007.

[13] A.E. Bernardini, O. Bertolami, Phys. Rev. D 77 (2008) 085032.

[14] B. Altschul, J. Phys. Conf. Ser. 173 (2009) 012003.

[15] S. Hollenberg, O. Micu, H. Päs, Phys. Rev. D 80 (2009) 053010.

[16] S. Ando, M. Kamionkowski, I. Mocioiu, Phys. Rev. D 80 (2009) 123522.

[17] M. Bustamante, A.M. Gago, C. Peña-Garay, J. Phys. Conf. Ser. 171 (2009) 012048

[18] P. Arias, J. Gamboa, Int. J. Mod. Phys. A 25 (2010) 277.

[19] S. Yang, B.-Q. Ma, Int. J. Mod. Phys. A 24 (2009) 5861.

[20] D.M. Mattingly, et al., JCAP 1002 (2010) 007.

[21] A. Bhattacharya, et al., JCAP 1009 (2010) 009.

[22] C.M. Ho, arXiv:1012.1053.

[23] J.S. Díaz, et al., Phys. Rev. D 80 (2009) 076007; V.A. Kostelecký, M. Mewes, Phys. Rev. D 70 (2004) 076002.

[24] L.B. Auerbach, et al., Phys. Rev. D 72 (2005) 076004.

[25] T. Katori, arXiv:1008.0906.

[26] P. Adamson, et al., Phys. Rev. Lett. 101 (2008) 151601.

[27] P. Adamson, et al., Phys. Rev. Lett. 105 (2010) 151601.

[28] R. Abbasi, et al., Phys. Rev. D 82 (2010) 112003.

[29] V.A. Kostelecký, M. Mewes, Phys. Rev. D 80 (2009) 015020; V.A. Kostelecký, M. Mewes, in preparation.

[30] V.A. Kostelecký, S. Samuel, Phys. Rev. D 39 (1989) 683; V.A. Kostelecký, R. Potting, Nucl. Phys. B 359 (1991) 545.

[31] S. Abe, et al., Phys. Rev. Lett. 100 (2008) 221803.

[32] P.F. Harrison, D.H. Perkins, W.G. Scott, Phys. Lett. B 530 (2002) 167.

[33] G. Bellini, et al., Phys. Rev. D 82 (2010) 033006.

[34] M.H. Ahn, et al., Phys. Rev. Lett. 90 (2003) 041801; Y. Ashie, et al., Phys. Rev. Lett. 93 (2004) 101801; D.G. Michael, et al., Phys. Rev. Lett. 97 (2006) 191801.

[35] V.A. Kostelecký, Phys. Rev. Lett. 80 (1998) 1818

[36] T. Katori, et al., Phys. Rev. D 74 (2006) 105009.

[37] J.K. Ahn, et al., arXiv:1003.1391; X. Guo, et al., hep-ex/0701029; F. Ardellier, et al., hep-ex/0606025.

[38] Y. Itow, et al., hep-ex/0106019; V. Barger, et al., arXiv:0705.4396; D.S. Ayres, et al., hep-ex/0503053.

[39] J. Alonso, et al., arXiv:1006.0260.

[40] G.T. Garvey, et al., Phys. Rev. D 72 (2005) 092001. 